\title{
The asymptotic price of anarchy for $k$-uniform congestion games
}

\author{
Jasper de Jong, Walter Kern, Berend Steenhuisen, and Marc Uetz \\ Faculty of Electrical Engineering, Mathematics and Computer Science, \\ University of Twente, P.O.Box 217, NL-7500 AE Enschede \\ $\{j \cdot$ dejong-3,w.kern, m. uetz $\}$ @utwente.nl \\ b.a.steenhuisen@student.utwente.nl
}

\begin{abstract}
We consider the atomic version of congestion games with affine cost functions, and analyze the quality of worst case Nash equilibria when the strategy spaces of the players are the set of bases of a $k$-uniform matroid. In this setting, for some parameter $k$, each player is to choose $k$ out of a finite set of resources, and the cost of a player for choosing a resource depends affine linearly on the number of players choosing the same resource. Earlier work shows that the price of anarchy for this class of games is larger than 1.34 but at most 2.15. We determine a tight bound on the asymptotic price of anarchy equal to $\approx 1.35188$. Here, asymptotic refers to the fact that the bound holds for all instances with sufficiently many players. In particular, the asymptotic price of anarchy is bounded away from $4 / 3$. Our analysis also yields an upper bound on the price of anarchy $<1.4131$, for all instances.
\end{abstract}

Keywords: congestion games, uniform matroid, asymptotic price of anarchy

\section{Introduction}

The effect of selfish behaviour on the overall system performance is a fundamental problem in the analysis of traffic networks, and more generally congestion games since the early works of Pigou [13], Wardrop [17] and Braess [4]. The problem also lies at the foundation of algorithmic game theory via Roughgarden and Tardos's analysis of equilibria in network routing games [16].

In network routing games, players have routing demands in a network and interact with each other by sharing network links with load-dependent costs. A Wardrop (or Nash) equilibrium is a routing of the demands such that no player can decrease her cost by unilaterally deviating. It is well known that equilibrium solutions may cause higher total costs than the system optimum, defined as the solution that minimizes the total cost of all players. If the cost functions are affine, Pigou's simple example shows that equilibrium solutions can exceed the cost of the system optimum by a factor as large as $4 / 3$ [13]. Pigou's example is surprisingly simple, as it consists of only two players, each of which can choose one of two parallel links, with constant and linear cost functions, respectively. 
Roughgarden and Tardos then showed that the relative gap can be at most $4 / 3$ in the worst case, for any network routing game with affine costs. The ratio between the total cost of a worst equilibrium solution and the minimum total cost has been named the price of anarchy (PoA) by Koutsoupias and Papadimitriou [11]. As to the PoA in network routing games, Roughgarden [15] also showed that the PoA is independent of the network topology for network routing games, as it is always attained on simple, Pigou-style networks.

In Wardrop's model, as well as in [16] and [15], the demand of any player may be distributed arbitrarily on minimum cost paths. A discrete version of Wardrop's model is that of a network routing game with unsplittable demands, also referred to as atomic network routing games. It has been introduced in its most general form by Rosenthal [14], who proved the existence of pure strategy Nash equilibria via potential functions, now often referred to as Rosenthal potential functions.

This type of atomic congestion games with affine cost functions are addressed in this paper. There is a finite set of players, a finite set of resources with affine cost functions, and a strategy of each player is to choose a set of resources from a given collection of subsets of the resources feasible for that player. In network routing games, this strategy space of a player is the set of all paths from a players' origin to destination, but in general it could be any set system specific to that player. When the strategy spaces of all players are identical, this is referred to as symmetric congestion games. Without any further restrictions on the strategy spaces of the players, the price of anarchy for affine cost functions is larger than $4 / 3$, namely $5 / 2$, as shown by Christodoulou and Koutsoupias [6] and Awerbuch et al. [1]. This price of anarchy is already attained for a network routing game with only three players, yet asymmetric strategy spaces. Correa et al. [7] eventually gave a class of instances of a symmetric, affine network routing game with price of anarchy asymptotically equal to $5 / 2$ (when the number of players tends to infinity).

In this context, an obvious question to ask is if restrictions on the strategy spaces of the players allows a substantial improvement of the price of anarchy bound of $5 / 2$. That this is generally possible is well known. For example, for instances where players choose only a singleton resource, and with symmetric strategy spaces, Lücking et al. [12] and Fotakis [10] showed that the price of anarchy is only $4 / 3$. However, if players choose a singleton resource but the strategy spaces are no longer symmetric, there is again an asymptotic lower bound $5 / 2$ (when the number of players tends to infinity) [5].

Our Contribution. The results discussed so far imply in particular that for atomic congestion games with affine cost functions, the price of anarchy does depend on the combinatorial structure of players' strategy spaces. Moreover, in light of the lower bound of [5] for asymmetric, singleton congestion games, there is room for improvement only for symmetric strategy spaces. Therefore, in [8] the case of $k$-uniform matroid congestion games with affine cost functions was studied. Here, each player is allowed to choose any of the $k$-element subsets of resources. They were able to show that the price of anarchy lies strictly in 
between 1.34 and 2.15. The present paper continues this line of research, and asks what the price of anarchy is if the number of players grows large. We answer this question, and prove that the asymptotic price of anarchy for $k$-uniform, affine congestion games equals $\approx 1.35188$. The interpretation of our asymptotic PoA bound is that it holds for any $k$ and any instance with a sufficient number of players. Tightness of our analysis follows by a matching lower bound, obtained through a parametric set of instances for which the price of anarchy exactly matches this bound. Our analysis also allows us to obtain an upper bound on the price of anarchy for any instance with any finite number of players. The bound we obtain here is slightly larger, yet $<1.4131$. That means that a finite number of instances may exist with a price of anarchy larger than 1.35189 , but never as large as 1.4131.

Note that the idea to study the asymptotic price of anarchy is not new. For instance, recent work on nonatomic network routing games considers the limit that the total demand grows large, referred to as a "high congestion regime" [3]. They show for example in the parallel link setting and under some regularity conditions on the cost functions, that the price of anarchy converges to 1 . See also [2] for further generalizations of these results. Another viewpoint on the asymptotics of the price of anarchy was taken by Feldman et al. [9]. Their work implies that for affine network routing games, the price of anarchy of atomic games converges to the $4 / 3$ bound of nonatomic games when the number of players grows large. Our result shows that $k$-uniform strategy spaces do not share this $4 / 3$ limit.

The main technical ingredient to obtain our results on the asymptotic price of anarchy is to interpret the solutions to $k$-uniform congestion games as $k$ matchings, and by observing that the symmetric difference between equilibrium and optimum solutions is the union of disjoint alternating paths. The bound is then obtained by carefully analysing the cost differences that these paths represent. We are convinced that this new way of analysing the problem might prove to be valuable also for other, more general problems.

\section{Preliminaries and notation}

An instance of the affine $k$-uniform congestion game is given by a parameter $k \in \mathbb{N}$, a complete bipartite graph $G=(N \cup R, E)$ and non-negative affine cost functions (i.e., functions of type $a x+b$ with $a, b \in \mathbb{R}_{+}$) $c_{r}$ for each $r \in R$. We interpret $N$ as a set of players and $R$ as a set of resources. Each player $i \in N$ has to pick a prescribed number $k$ of resources in $R$. We refer to $|N|+|R|$ as the size of the instance.

It is natural to model this situation in the context of matchings. Each player is to choose a set of $k$ resources $r \in R$ or, equivalently, a set of $k$ incident edges. Slightly misusing the standard notation, let us define a $k$-matching to be a subset $M \subseteq E$ whose degree vector $d=d^{M}$ satisfies $d_{i}=k$ for all $i \in N$. Each such $k$-matching induces corresponding $\operatorname{costs} c_{r}^{M}=c_{r}\left(d_{r}\right), r \in R$. Player $i \in N$ experiences corresponding $\operatorname{cost} C_{i}=C_{i}^{M}:=\sum_{i r \in M} c_{r}^{M}$ and we define the total 
cost to be $c^{M}:=\sum_{i} C_{i}^{M}=\sum_{R} d_{r} c_{r}\left(d_{r}\right)$. Let $O P T \subseteq E$ denote a $k$-matching that minimizes the total cost. Sometimes it is also convenient to work with edge costs defined by $c_{i r}=c_{i r}^{M}:=c_{r}^{M}$. Thus $C_{i}$ then equals the total cost of the edges in $M$ that are incident to $i$.

Given a $k$-matching $M \subseteq E$ and a player $i \in N$, we let $M(i) \subseteq R$ denote the set of resources assigned to $i$. Similarly, for $r \in R$ we let $M(r) \subseteq N$ denote the set of players that use resource $r$. A $k$-matching $M \subseteq E$ is called a Nash equilibrium if each player $i \in N$ is satisfied with her set $M(i) \subseteq R$ in the sense that no other choice would strictly decrease her cost $C_{i}$ (assuming that the remaining sets $M(j)$ stay unchanged). It is straightforward to verify that this condition is satisfied iff $C_{i}$ cannot be decreased by exchanging a single resource, i.e., switching from $M(i)$ to $M^{\prime}(i)=M(i)-r+s$ for some $r \in M(i), s \notin M(i)$ (thereby increasing the degree of $s$ to $d_{s}^{M}+1$ ). In other words, Nash equilibria are characterized by the Nash condition: For all $i \in N$,

$$
\text { ir } \in M, i s \notin M \Rightarrow c_{s}\left(d_{s}^{M}+1\right) \geq c_{r}^{M}\left(d_{r}\right) .
$$

The costs $c_{s}\left(d_{s}^{M}+1\right)$ that a player experiences when he replaces one of his current resources $r$ by $s$ is also called the opportunity cost of $s$.

In what follows we assume that $N E \subseteq E$ is a Nash equilibrium. To simplify the notation, we let $d^{*}$ and $\bar{d}$ denote the degree vectors of $O P T$ and $N E$, resp. To simplify even more, let us denote the Nash costs by $\bar{c}_{r}:=c_{r}\left(\bar{d}_{r}\right)$ and the corresponding opportunity costs by $\bar{c}_{r}^{+}:=c_{r}\left(\bar{d}_{r}+1\right)$. So the Nash condition simply reads as

$$
\text { ir } \in N E, i s \notin N E \Rightarrow \bar{c}_{s}^{+} \geq \bar{c}_{r} .
$$

We seek to bound the $\mathrm{PoA}=c^{N E} / c^{O P T}$. Our main result is

Theorem 1. Any sequence of instances $\left(k_{t}, G_{t}=\left(N_{t}, R_{t}\right)\right)$ with an increasing number of players $\left|N_{t}\right| \rightarrow \infty$ has $\lim \sup c^{N E} / c^{O P T}<1.35189$.

Section 3 provides some simplifications and "without loss of generality" assumptions. In Section 4 we upper bound the gap $c^{N E}-c^{O P T}$ and in Section 5 we lower bound the Nash cost $c^{N E}$. Together, these two results prove $\limsup c^{N E} / c^{O P T} \leq 1.35188 \ldots$ Corresponding tight instances (with $|N|, k \rightarrow$ $\infty)$ are presented in Section 6 . We conclude with some remarks and open problems in Section 7.

\section{Simplifications of worst-case instances}

As in the previous section, we consider an instance of the affine k-uniform congestion game of size $m=|N|+|R|$ with cost minimal $k$-matching $O P T \subseteq E$ and Nash equilibrium $N E \subseteq E$. As before, we denote by $d^{*}$ and $\bar{d}$ the degree vectors of $O P T$ and $N E$, resp. Let $\rho=c^{N E} / c^{O P T}$ be the PoA of the instance. We call the instance $k$-critical (or critical for short) if no instance of size $<m$ has PoA 
$\geq \rho$ and no instance of size $=m$ has PoA $>\rho$. Clearly, in order to upper bound the PoA, we may restrict our attention to critical instances. In the following, we will derive some helpful properties of critical instances. Pigou's classic example is obviously a critical instance of size 4 . Hence, a critical instance of size $\geq 5$ must have $\mathrm{PoA}>4 / 3$.

$O P T$ and $N E$ induce a partition of $R$ into three sets:

$$
\begin{array}{ll}
O:=\left\{o \in R \mid \bar{d}_{o}>d_{o}^{*}\right\} & \text { ("overloaded resources") } \\
U:=\left\{u \in R \mid \bar{d}_{u}<d_{u}^{*}\right\} & \text { ("underloaded resources") } \\
B:=\left\{b \in R \mid \bar{d}_{b}=d_{b}^{*}\right\} & \text { ("balanced resources") }
\end{array}
$$

The symmetric difference $O P T \oplus N E$ consists of exclusive Nash edges e $\in$ $N E \backslash O P T$ and exclusive $O P T$ edges $e \in O P T \backslash N E$. The set $O P T \oplus N E$ can be partitioned into a set $\mathcal{P}$ of pairwise edge disjoint alternating paths joining $O$ to $U$ and a set $\mathcal{C}$ of alternating (even) cycles. (Here, "alternating" means that consecutive edges alternate between exclusive OPT and exclusive Nash edges.) We may assume w.l.o.g. that $\mathcal{C}=\emptyset$ : If $C \in \mathcal{C}$, then $O P T \oplus C$ is obviously also cost minimal (as the $d_{r}^{*}$ remain unchanged). We may continue this process of "switching along alternating cycles" until no one is left.

Another w.l.o.g. assumption is the following

Lemma 1. We may assume that all cost functions $c_{r}$ for $r \in O \cup B$ are linear, i.e., $c_{r}(x)=c_{r} \cdot x$ for some constant $c_{r} \geq 0$.

Proof. Assume to the contrary that $c_{r}(x)=a x+b$ with $b>0$. Define $\tilde{c}_{r}(x):=$ $x \cdot \bar{c}_{r} / \bar{d}_{r}$ and observe that $\tilde{c}_{r}\left(\bar{d}_{r}\right)=\bar{c}_{r}$ and $\tilde{c}\left(\bar{d}_{r}+1\right)>\bar{c}_{r}^{+}$. This implies that any Nash equilibrium remains Nash w.r.t. the modified cost functions and $c^{N E}$ is unchanged. Furthermore, $\tilde{c}\left(d_{r}^{*}\right) \leq c_{r}\left(d_{r}^{*}\right)$, since $d_{r}^{*} \leq \bar{d}_{r}$. Thus $c^{O P T}$ can only decrease.

The gap $c^{N E}-c^{O P T}$ can be best understood by observing how the cost increases while we move from $O P T$ to $N E$ by switching along alternating paths $P \in \mathcal{P}$. Let $\mathcal{P}_{o} \subseteq \mathcal{P}$ denote the set of paths $P \in \mathcal{P}$ that start in $o \in O$. So $\left|\mathcal{P}_{o}\right|=\bar{d}_{o}-d_{o}^{*}$. Switching $O P T$ and $N E$ along all paths in $P_{o}$ simultaneously has the following effect on the edge costs of $P=(o, i, \ldots, j, u) \in \mathcal{P}_{o}$ : Edge io with cost $\bar{c}_{o}$ enters and edge $j u$ with cost $c_{u} \geq \bar{c}_{u}^{+}$leaves the current $k$-matching. Apart from that, switching $O P T$ and $N E$ along $P$ does not affect any costs of intermediate resources $r \neq o, u$ that $P$ may visit. Thus, when passing from $O P T$ to $N E$, the edges of $P$ contribute a total increase of $\bar{c}_{o}-c_{u} \leq \bar{c}_{o}-\bar{c}_{u}^{+}$. We refer to the latter as the internal cost bound of path $P$. In addition to these $\bar{d}_{o}-d_{o}^{*}$ internal path costs, there are external costs experienced by $d_{o}^{*}$ edges whose edge costs are raised from $c_{o} \cdot d_{o}^{*}$ to $c_{o} \cdot \bar{d}_{o}$, due to the increase in degree of $o$.

Summarizing, the gap can be bounded by

$$
c^{N E}-c^{O P T} \leq \sum_{P=(o, \ldots, u) \in \mathcal{P}} \bar{c}_{0}-\bar{c}_{u}^{+}+\sum_{O} d_{o}^{*}\left(\bar{d}_{o}-d_{o}^{*}\right) c_{o}
$$


The external part can be upper bounded by $\frac{1}{4} \sum_{O} \bar{d}_{o}^{2} c_{o} \leq \frac{1}{4} c^{N E}$ (the maximum being attained for $d_{o}^{*}=\bar{d}_{o} / 2$ ). Thus, if the internal part is close to zero, we would get a $\mathrm{PoA} \approx 4 / 3$. The difficult part is to estimate the internal cost bounds $\bar{c}_{o}-\bar{c}_{u}^{+}$. To provide some intuition, we observe the following simple fact:

Lemma 2. Each $P=(o, i, \ldots, j, u) \in \mathcal{P}$ with positive internal cost bound $\bar{c}_{o}-$ $\bar{c}_{u}^{+}>0$ has length at least 4 .

Proof. Being an alternating path that starts in a Nash edge $i o$ and ends in the $O P T$ edge $j u, P$ must have even length. If $P$ had length 2 , i.e., $P=(o, i, u)$, with $i o \in N E$ and $i u \notin N E$, then $\bar{c}_{o}-\bar{c}_{u}^{+}>0$ would contradict the Nash condition (1).

Thus each path that contributes a positive amount to the gap also "increases" the total Nash cost $c^{N E}$. (There must be at least a second Nash edge $e \in N E$ on $P$, apart from the first edge $i o$.) Considerations of this kind in Sections 4 and 5 will allow us to bound the gap in terms of $c^{N E}$, i.e., to bound the relative gap $\left(c^{N E}-c^{O P T}\right) / c^{N E}$, which then yields a corresponding bound for $c^{N E} / c^{O P T}$. As a little exercise, consider the following

Lemma 3. The Pigou example of size 4 is (up to scaling) the only critical instance for $k=1$. Thus $P o A \leq 4 / 3$ for $k=1$.

Proof. Consider any path $P=(o, i, \ldots, u) \in \mathcal{P}$. Then $i o \in N E$, implying that $i u \notin N E$ (since $k=1)$. Hence $\bar{c}_{o}-\bar{c}_{u}^{+} \leq 0$ follows from the Nash property. Then (2) yields $c^{N E}-c^{O P T} \leq \sum_{O} d_{o}^{*}\left(\bar{d}_{o}-d_{o}^{*}\right) c_{o} \leq \frac{1}{4} c^{N E}$, i.e., $c^{N E} / c^{O P T} \leq 4 / 3$, as in Pigou's example. Criticality then implies that the instance has size 4 , and it is then straightforward to show that (up to scaling) only Pigou's example achieves this bound.

Thus we are left to deal with the case $k \geq 2$. Obviously, for $k \geq 2$, a $k$-critical instance with $\mathrm{PoA}>4 / 3$ must have at least size 5 (three resources) by definition of criticality. We end this section with some more properties of such instances, which were already shown in [8].

Lemma 4 ([8]). For $k \geq 2$, no $k$-critical instance has $\bar{d}_{r}=|N|$ for some $r \in R$.

The following result helps to bound the internal part in equation (2). Let $c_{O}^{\max }:=$ $\max \left\{\bar{c}_{o} \mid o \in O\right\}$.

Lemma 5 ([8]). In a critical instance, $\bar{c}_{r}^{+} \geq c_{O}^{\max } / 2$ for all $r \in R$. Hence $\bar{c}_{o}-\bar{c}_{u}^{+} \leq \bar{c}_{o} / 2$ for all $o \in O, u \in U$.

Note that, as the cost functions are affine, Lemma 5 also implies $\bar{c}_{r} \geq \frac{1}{2} \bar{c}_{r}^{+} \geq$ $\frac{1}{4} c_{O}^{\max }$ for all $r \in R$. 


\section{An upper bound on the relative gap}

We order the paths $P=(o, \ldots, u) \in \mathcal{P}$ according to both non-decreasing values of $\bar{c}_{o}$ as well as non-increasing values of $\bar{c}_{u}^{+}$. Let $\Delta:=|\mathcal{P}|$ and assume $P_{1}, \ldots, P_{\Delta}$ where $P_{t}=\left(o_{t}, \ldots, u_{t}\right)$ is an ordering with $\bar{c}_{o_{1}} \leq \ldots \leq \bar{c}_{o_{\Delta}}$ and let $P_{1}^{\prime}, \ldots, P_{\Delta}^{\prime}$ with $P_{t}^{\prime}=\left(o_{t}^{\prime}, \ldots, u_{t}^{\prime}\right)$ satisfy $\bar{c}_{u_{1}^{\prime}}^{+} \geq \ldots \geq \bar{c}_{u_{\Delta}^{\prime}}^{+}$.

In case $\bar{c}_{o_{\Delta}} \leq \bar{c}_{u_{\Delta}^{\prime}}^{+}$, all paths have internal cost bound $\bar{c}_{o}-\bar{c}_{u}^{+} \leq \bar{c}_{o_{\Delta}}-\bar{c}_{u_{\Delta}^{\prime}}^{+} \leq 0$, so the $\mathrm{PoA}$ is no more than $4 / 3$ (cf. equation (2)). So the interesting (critical) case is when $\bar{c}_{o_{\Delta}}>\bar{c}_{u_{\Delta}^{\prime}}^{+}$. Let $t \geq 0$ be the first index for which $\bar{c}_{o_{t+1}}>\bar{c}_{u_{t+1}^{\prime}}^{+}$. Let

$$
\mathcal{P}^{-+}:=\left\{P_{1}, \ldots, P_{t}\right\} \cap\left\{P_{1}^{\prime}, \ldots, P_{t}^{\prime}\right\} .
$$

Loosely speaking, one might say that $\mathcal{P}^{-+}$consists of those paths in $\mathcal{P}$ that join some $o \in\left\{o_{1}, \ldots, o_{t}\right\}$ to some $u \in\left\{u_{1}^{\prime}, \ldots, u_{t}^{\prime}\right\}$. (Here, we slightly misuse the notation, as, e.g., $\left\{o_{1}, \ldots, o_{t}\right\}$ is actually a multiset whose elements may also appear among $o_{t+1}, \ldots, o_{\Delta}$.) Thus each $P \in \mathcal{P}^{-+}$has internal cost bound $\bar{c}_{o}-\bar{c}_{u}^{+} \leq 0$. Similarly, each path in

$$
\mathcal{P}^{+-}:=\left\{P_{t+1}, \ldots, P_{\Delta}\right\} \cap\left\{P_{t+1}^{\prime}, \ldots, P_{\Delta}^{\prime}\right\}
$$

has internal cost bound $\bar{c}_{o}-\bar{c}_{u}^{+}>0$. The remaining paths in

$$
\begin{aligned}
& \mathcal{P}^{++}:=\left\{P_{t+1}, \ldots, P_{\Delta}\right\} \cap\left\{P_{1}^{\prime}, \ldots, P_{t}^{\prime}\right\} \text { and } \\
& \mathcal{P}^{--}:=\left\{P_{1}, \ldots, P_{t}\right\} \cap\left\{P_{t+1}^{\prime}, \ldots, P_{\Delta}^{\prime}\right\}
\end{aligned}
$$

may have positive or negative internal cost bounds. Figure 1 illustrates the idea behind these definitions.

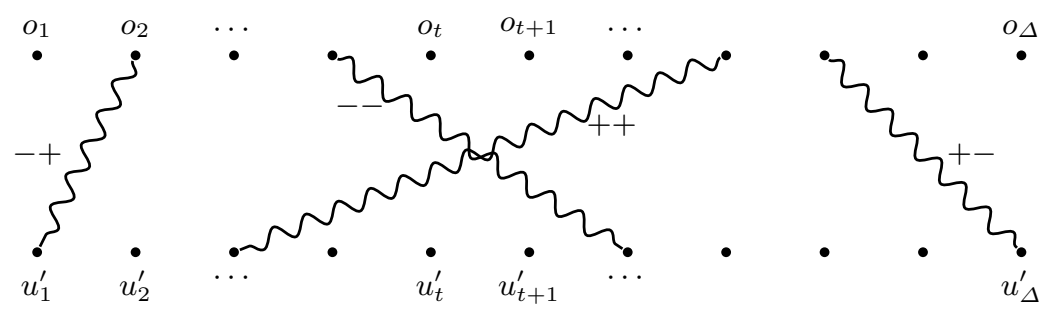

Fig. 1. Possible augmenting paths in $\mathcal{P}^{-+}, \mathcal{P}^{--}, \mathcal{P}^{++}$, and $\mathcal{P}^{+-}$, respectively.

In any case, however, $P=(o, \ldots, u) \in \mathcal{P}^{++}$has internal cost bound $\bar{c}_{o}-$ $\bar{c}_{u}^{+} \leq \bar{c}_{o}-\bar{c}_{u_{t}}^{+}$and any $P^{\prime}=\left(o^{\prime}, \ldots, u^{\prime}\right) \in \mathcal{P}^{--}$has internal cost $\bar{c}_{o}-\bar{c}_{u^{\prime}}^{+} \leq$ $\bar{c}_{o_{t}}-\bar{c}_{u^{\prime}}^{+} \leq \bar{c}_{u_{t}}^{+}-\bar{c}_{u^{\prime}}^{+}$. Thus the internal costs of such $P$ and $P^{\prime}$ add to at most $\bar{c}_{o}-\bar{c}_{u^{\prime}}^{+} \leq \bar{c}_{o} / 2\left(c f\right.$. Lemma 5). Since $\left|\mathcal{P}^{++}\right|=\left|\mathcal{P}^{--}\right|\left(=\Delta-t-\left|\mathcal{P}^{+-}\right|\right)$, we may group the paths $\mathcal{P}^{++} \cup \mathcal{P}^{--}$in pairs $P \in \mathcal{P}^{++}, P^{\prime} \in \mathcal{P}^{--}$with total internal 
cost at most $\bar{c}_{o} / 2$, where $o$ is the starting point of $P$. Similarly, each (single) path $P=(o, \ldots, u) \in \mathcal{P}^{+-}$contributes $\bar{c}_{o}-\bar{c}_{u}^{+} \leq \bar{c}_{o} / 2$ to the internal costs. Thus if we let $O^{+} \subseteq O$ denote the set of resources that appear among $o_{t+1}, \ldots, o_{\Delta}$, let $\mathcal{P}^{+}:=\mathcal{P}^{+-} \cup \mathcal{P}^{++}$, let $\mathcal{P}_{o}^{+}$denote the paths in $\mathcal{P}^{+}$that start in $o$, and write $\Delta_{o}^{+}:=\left|\mathcal{P}_{o}^{+}\right|$, the gap is bounded by

$$
c^{N E}-c^{O P T} \leq \sum_{O^{+}} \Delta_{o}^{+} \bar{c}_{o} / 2+\sum_{O} d_{o}^{*}\left(\bar{d}_{o}-d_{o}^{*}\right) c_{o}
$$

For further use we also introduce the corresponding set $U^{-} \subseteq U$ of resources that appear among $\left\{u_{t+1}^{\prime}, \ldots, u_{\Delta}^{\prime}\right\}$ and, for each $u \in U^{-}$the set $\mathcal{P}^{-}:=\mathcal{P}^{--} \cup \mathcal{P}^{+-}$, $\mathcal{P}_{u}^{-}$the paths in $\mathcal{P}^{-}$that end in $u$ along with the corresponding $\Delta_{u}^{-}:=\left|\mathcal{P}_{u}^{-}\right|$. Note that $\sum_{U^{-}} \Delta_{u}^{-}=\sum_{O^{+}} \Delta_{o}^{+}=\Delta-t$. Furthermore, $\bar{c}_{o}>\bar{c}_{u}^{+}$holds for all $o \in O^{+}, u \in U^{-}$.

Remark. As we will see in Section 6, tight examples for the PoA $=1.35188 \ldots$ are obtained with $O=O^{+}$(and $U=U^{-}, \mathcal{P}=\mathcal{P}^{+-}$).

\section{Lower bounding the cost of a Nash equilibrium $c^{N E}$}

As mentioned earlier, we seek to bound the relative gap $\left(c^{N E}-c^{O P T}\right) / c^{N E}$ which then yields a corresponding upper bound for the PoA. To this end, we have to lower bound $c^{N E}$, which is the purpose of this section. We first lower bound the number of edges in $N E$ and afterwards deal with their costs.

Let $I^{+}:=N E\left(O^{+}\right)$denote the set of players $i \in N$ that are joined to $O^{+}$ by a Nash edge. As $\bar{c}_{o}>\bar{c}_{u}^{+}$for all $o \in O^{+}, u \in U^{-}$, the Nash property implies $i u \in N E$ for all $i \in I^{+}, u \in U^{-}$. Thus we obtain

$$
\left|I^{+}\right| \geq \sum_{O^{+}} \bar{d}_{o} /\left(k-\left|U^{-}\right|\right),
$$

since each $i \in I^{+}$can receive at most $k-\left|U^{-}\right|$Nash edges from $O^{+}$(in addition to its $\left|U^{-}\right|$Nash edges from $\left.U^{-}\right)$.

Let $J:=\left\{j \in N \mid \exists P=(o, \ldots, j, u) \in \mathcal{P}^{-}\right\}$be the set of "last players" on paths in $\mathcal{P}^{-}$. By definition of $\mathcal{P}^{-}$, the last edge $j u$ of a path in $\mathcal{P}^{-}$joins $j$ to $U^{-}$. Hence at most $\left|U^{-}\right|$paths in $\mathcal{P}^{-}$can go through a fixed $j \in J$. There are $\Delta-t=\sum_{O^{+}} \Delta_{o}^{+}$paths in $\mathcal{P}^{-}$. This implies

$$
|J| \geq \sum_{U^{-}} \Delta_{u}^{-} /\left|U^{-}\right|=\sum_{O^{+}} \Delta_{o}^{+} /\left|U^{-}\right|
$$

Next, we observe that

$$
I^{+} \cap J=\emptyset .
$$

Indeed, if $i \in I^{+} \cap J$, then $i o \in N E$ for some $o \in O^{+}$and $i u \in O P T \backslash N E$ for some $u \in U^{-}$, contradicting the Nash property (as $\bar{c}_{o}>\bar{c}_{u}^{+}$). Hence we can 
estimate the number of edges (due to (6) without any double counting):

$$
|N E| \geq \sum_{O^{+}} \bar{d}_{o}+\left|I^{+}\right|\left|U^{-}\right|+|J| k .
$$

To estimate the corresponding edge costs is a bit more involved: First, observe that if $\sum_{O^{+}} \bar{d}_{o} \leq \epsilon \sum_{R} \bar{d}_{r}$ for a sufficiently small $\epsilon>0$ (say, $\epsilon=0.001$ is certainly sufficient), then we deduce from equation (3) and Lemma 5 (implying $\left.\bar{c}_{r} \geq \frac{1}{4} c_{O}^{\max }\right)$ that

$$
c^{N E}-c^{O P T} \leq \epsilon \sum_{R} \bar{d}_{r}^{2} c_{O}^{\max }+\frac{1}{4} \sum_{O} \bar{d}_{o}^{2} c_{o} \leq 4 \epsilon \sum_{R} \bar{d}_{r} \bar{c}_{r}+\frac{1}{4} c^{N E},
$$

so the instance has a gap close to $\frac{1}{4} c^{N E}$, corresponding to, say, a PoA $\leq 1.34$ thus an uninteresting (uncritical) instance in view of Section 6 below.

Thus, in what follows, we restrict ourselves to instances where $\sum_{O^{+}} \bar{d}_{o} \geq$ $\epsilon \sum_{R} \bar{d}_{r}=\epsilon k|N|$ for some small $\epsilon>0$. Since we seek to analyze instances with $|N| \rightarrow \infty$, we may thus assume that $\left(\sum_{O^{+}} \bar{d}_{o}\right) / k \rightarrow \infty$. Then also $\left|I^{+}\right| \rightarrow \infty(c f$. equation (4)). This further implies $\bar{d}_{u} \rightarrow \infty$ and, consequently (using Lemma 5), $\bar{c}_{u} \geq c_{O}^{\max } / 2$ in the limit for $u \in U^{-}$. Therefore, in our estimate of $c^{N E}$ below, we let each $u \in U^{-}$have $\bar{c}_{u}=c_{O}^{\max } / 2$.

Next we deal with the costs of the $k|J|$ Nash edges incident to $J$. We intend to show that each of these edges also has Nash cost $\geq c_{O}^{\max } / 2$, or, at least, that we can account such an amount for each of these edges. Let $j r \in N E$ be any such edge. Pick any $o \in O^{+}$with $\bar{c}_{o}=c_{O}^{\max }$ and any $i \in I^{+}$with $i o \in N E$. If $i r \notin N E$, then, by the Nash condition, we have $\bar{c}_{r}^{+} \geq c_{O}^{\max }$, implying $\bar{c}_{r} \geq c_{O}^{\max } / 2$, and we are done. Otherwise, suppose $i r \in N E$. Assume first that $j r$ is the only Nash edge from $J$ to $r$. Then $\bar{d}_{r} \geq 2$ (one edge from $i$, one from $j$ ), hence $\bar{c}_{r} \geq c_{O}^{\max } / 3$ (using $\bar{c}_{r} \geq \frac{2}{3} \bar{c}_{r}^{+}$and Lemma 5). In this case we discharge the cost of $i r$ to $j r$, resulting in an accounted cost of $\frac{2}{3} c_{O}^{\max }$ on the edge $j r$. In general, if there are $d \geq 1$ edges joining $J$ to $r$, each of these has already Nash cost $\bar{c}_{r} \geq \frac{d+1}{d+2} \bar{c}_{r}^{+} \geq \frac{d+1}{d+2} c_{O}^{\max } / 2$. (Note that, together with the edge $i r$, there are at least $d+1$ Nash edges incident to $r$.) The edge $i r \in N E$ has the same Nash cost $\bar{c}_{r} \geq \frac{d+1}{d+2} c_{O}^{\max } / 2$. Thus if we discharge a fraction $\frac{1}{d}$ of its cost to each of the edges $j r \in N E$, then each of the latter gets an accounted total cost of $\geq\left(\frac{d+1}{d+2}+\frac{d+1}{d(d+2)}\right) c_{O}^{\max } / 2 \geq c_{O}^{\max } / 2$.

Accounting $\frac{1}{2} c_{O}^{\max }$ for all edges $j r \in J \times R$ and $i u \in I^{+} \times U^{-}$, we may estimate the relative gap as follows (abbreviating $u^{-}:=\left|U^{-}\right|$):

$$
\left(c^{N E}-c^{O P T}\right) / c^{N E} \leq \frac{\sum_{O^{+}} \Delta_{o}^{+} \bar{c}_{o} / 2+\sum_{O} d_{o}^{*}\left(\bar{d}_{o}-d_{o}^{*}\right) c_{o}}{\sum_{O} \bar{d}_{o}^{2} c_{o}+\left(\frac{u^{-}}{k-u^{-}} \sum_{O^{+}} \bar{d}_{o}+\frac{k}{u^{-}} \sum_{O^{+}} \Delta_{o}^{+}\right) c_{O}^{\max } / 2},
$$

The three sums in the denominator correspond to the cost of Nash edges in $N \times O, I^{+} \times U^{-}$, and $J \times R$, resp. Since $d_{o}^{*}\left(\bar{d}_{o}-d_{o}^{*}\right) \leq \frac{1}{4} \bar{d}_{o}^{2}$, removing the terms 
corresponding to $o \in O \backslash O^{+}$in both the numerator and the denominator in (9) can only increase the right hand side. After replacing $O$ by $O^{+}$in the two sums in (9), we may estimate the fraction by considering the corresponding fractions per $o \in O^{+}$:

$$
\begin{aligned}
\left(c^{N E}-c^{O P T}\right) / c^{N E} & \leq \max _{o \in O^{+}} \frac{\Delta_{o}^{+} \bar{c}_{o} / 2+d_{o}^{*}\left(\bar{d}_{o}-d_{o}^{*}\right) c_{o}}{\bar{d}_{o}^{2} c_{o}+\left(\frac{u^{-}}{k-u^{-}} \bar{d}_{o}+\frac{k}{u^{-}} \Delta_{o}^{+}\right) c_{O}^{\max } / 2} \\
& \leq \max _{o \in O^{+}} \frac{\Delta_{o}^{+} \bar{d}_{o} / 2+d_{o}^{*}\left(\bar{d}_{o}-d_{o}^{*}\right)}{\bar{d}_{o}^{2}+\left(\frac{u^{-}}{k-u^{-}} \bar{d}_{o}^{2}+\frac{k}{u^{-}} \Delta_{o}^{+}\right) / 2} .
\end{aligned}
$$

The latter inequality is obtained by replacing $c_{O}^{\max }$ with the smaller or equal $\bar{d}_{o} c_{o}$ and dividing by $c_{o}$. Now, fix any $o \in O^{+}$where the maximum is attained. Again, $d_{o}^{*}\left(\bar{d}_{o}-d_{o}^{*}\right) \leq \frac{1}{4}$ implies that the maximum is attained when $\Delta_{o}^{+}$is as large as possible (unless $k / u^{-}>4$, in which case the whole fraction is less than $\frac{1}{4}$ ). Thus we may assume $\Delta_{o}^{+}=\bar{d}_{o}-d_{o}^{*}$. Writing $\bar{d}_{o}=\beta d_{o}^{*}, k=\alpha u^{-}$(with $\alpha, \beta \geq 1$ ), the maximum in (10) can be bounded by

$$
\mu=\max _{\alpha, \beta} \frac{(\beta-1) \beta / 2+\beta-1}{\beta^{2}+\left(\beta^{2} /(\alpha-1)+(\beta-1) \beta \alpha\right) / 2}=0.260292 \ldots
$$

(according to WolframAlpha, for $\alpha \approx 2.3, \beta \approx 2.5$ ). The corresponding upper bound for $c^{N E} / c^{O P T}$ is $\rho=1 /(1-\mu) \leq 1.35188 \ldots$. This finishes the proof of Theorem 1.

\section{Tight lower bound construction}

We construct examples with $\mathrm{PoA}=1.35188 \ldots$ as suggested by the analysis in Sections 4 and 5. A close look reveals that a tight example must have $O=O^{+}$ (and, correspondingly, $U=U^{-}$) and $\bar{d}_{o}-\bar{d}_{o}^{*}$ many paths in $\mathcal{P}$ starting in $o \in O$. We thus define $N:=I \cup J$ and $R=O \cup B \cup U$. The number $|I|=|U| \in \mathbb{N}$ is a free parameter fixing the problem instance. (We thus let $|I| \rightarrow \infty$, which also increases the number of players to infinity.) In addition, we have the two parameters $\alpha \approx 2.3, \beta \approx 2.5$ as in Section 5. These determine $k:=\alpha|U|$ and $\bar{d}_{o}=\beta d_{o}^{*}$.

The cost functions are $c_{o}(x)=\frac{1}{|U|} x$ for $o \in O$. For $b \in B$ we have $c_{b}(x)=\frac{1}{2} x$ and for $u \in U$ we have constant costs $c_{u}(x)=1 / 2$.

Each $i \in I$ is joined by Nash edges to all nodes in $O$ and $U$ (and nothing else). Hence $k=\alpha|U|$ implies $|O|=(\alpha-1)|U|$. For each $i \in I$,all Nash edges to $U$ are also in $O P T$. In addition, a $\frac{1}{\beta}$ fraction of its Nash edges to $O$ is also in $O P T$. No other $O P T$ edges are incident to $O$. Assuming that we distribute the $O P T \cap N E$ edges from $I$ to $O$ in a regular manner, we may thus assume that each $o \in O$ receives $d_{o}^{*} O P T$ edges and $|U|=\bar{d}_{o}=\beta d_{o}^{*}$ Nash edges, as required. The Nash cost of $o \in O$ is thus $\bar{c}_{o}=|U| \frac{1}{|U|}=1$ and its $O P T$ cost is 
$c_{o}^{*}=\frac{|U|}{\beta} \frac{1}{|U|}=\frac{1}{\beta}$. Thus the total Nash cost of $O$ is $\bar{c}_{O}=|O| \bar{d}_{o} \bar{c}_{o}=(\alpha-1)|U||U|$, while the $O P T$ cost equals $c_{O}^{*}=|O| d_{o}^{*} c_{o}^{*}=(\alpha-1) \frac{|U|}{\beta}|U| \frac{1}{\beta}=(\alpha-1) / \beta^{2}|U|^{2}$.

Each $i \in I$ now still misses an amount of $k-\frac{|O|}{\beta}-|U|$ edges in $O P T$. These are exclusive $O P T$ edges leading to $|I|\left(k-\frac{|O|}{\beta}-|U|\right)$ resources $b \in B$, each of degree $\bar{d}_{b}=d_{b}^{*}=1$. We denote this set of resources $b$ by $B_{\mathcal{P}}$ to indicate that these are the balanced resources on alternating paths. Thus $\left|B_{\mathcal{P}}\right|=|I|(k-|O| / \beta-|U|)=$ $|U|(\alpha|U|-(\alpha-1) / \beta|U|-|U|)=(\alpha-1)(1-1 / \beta)|U|^{2}$.

Each $j \in J$ is joined to all of $U$ by exclusive $O P T$ edges. In addition, it is joined by $N E \cap O P T$ edges to $k-|U|$ nodes in $B \backslash B_{\mathcal{P}}$, each of which has $\bar{d}_{b}=d_{b}^{*}=1$. Now each $j \in J$ is still missing $|U|$ exclusive Nash edges. So we partition $B_{\mathcal{P}}$ into sets of size $|U|$ each and join each of these sets to some $j \in J$. This fixes the size $|J|:=\left|B_{\mathcal{P}}\right| /|U|=(\alpha-1)(1-1 / \beta)|U|$ and completes the description of our instance. Figure 2 illustrates this construction.

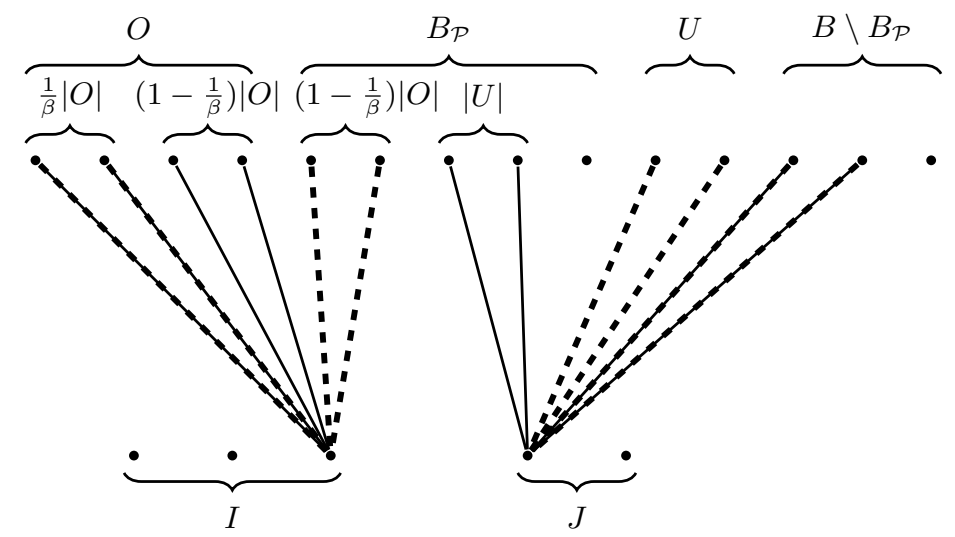

Fig. 2. Construction of a matching lower bound instance with $\mathrm{PoA} \approx 1.35188$. Edges in $O P T$ are dashed lines, while edges in the $N E$ are solid.

To finish, we need to calculate the remaining costs. For $u \in U$ we have constant $\operatorname{costs} c_{u}=1 / 2$, so the Nash costs are $\bar{c}_{U}=|I||U| \frac{1}{2}=|U|^{2} \frac{1}{2}$. The OPT costs are $c_{U}^{*}=(|J||U|+|I||U|) \frac{1}{2}=\left(\left|B_{\mathcal{P}}\right|+|U|^{2}\right) \frac{1}{2}=((\alpha-1)(1-1 / \beta)+1)|U|^{2} \frac{1}{2}$. Finally, the costs of $B$ are the same for both $N E$ and $O P T$. They equal $k|J| \frac{1}{2}=$ $\alpha(\alpha-1)(1-1 / \beta)|U|^{2} \frac{1}{2}$. 
Summarizing, according to WolframAlpha we obtain (dividing by $|U|^{2}$ ) with $\alpha \approx 2.3$ and $\beta \approx 2.5$

$$
\begin{aligned}
c^{N E} / c^{O P T} & =\frac{(\alpha-1)+\frac{1}{2}+\alpha(\alpha-1)(1-1 / \beta) \frac{1}{2}}{(\alpha-1) / \beta^{2}+(\alpha-1)\left(1-\frac{1}{\beta}\right) \frac{1}{2}+\frac{1}{2}+\alpha(\alpha-1)\left(1-\frac{1}{\beta}\right) \frac{1}{2}} \\
& =\frac{2+1 /(\alpha-1)+\alpha(1-1 / \beta)}{2 / \beta^{2}+1-1 / \beta+1 /(\alpha-1)+\alpha(1-1 / \beta)}=1.35188 \ldots
\end{aligned}
$$

\section{$7 \quad$ Remarks and open problems}

Of course the most natural open problem is to find out whether the price of anarchy equals $\approx 1.35188$ also for instances with only a few players. In our analysis in Section 5 we used the assumtion that $|N| \rightarrow \infty$ only in order to conclude that $\bar{c}_{u} \geq \frac{1}{2} c_{O}^{\max }$ in the limit. Without the assumption $|N| \rightarrow \infty$ we can still estimate $\bar{c}_{u} \geq \frac{1}{4} c_{O}^{\max }$ with the help of Lemma 5. Performing the same analysis with this weaker estimate still leads to a reasonably small PoA $<1.4131$ for all instances. Reconsidering the lower bound example in Section 5, we find that the PoA depends on the size of $I$ (linearly related to $|N|$ ) determining the $\bar{c}_{u}, u \in U$ and how well $\alpha, \beta$ are approximated (i.e., $|O|$ must be a multiple of $\beta$ ). If the parameters are well approximated for some small $|N|$ (implying small $|I|$ and hence $\bar{c}_{u}$ significantly less than $\frac{1}{2}$ ), it is conceivable that for these (finitely many) values of $|N|$ a $\mathrm{PoA}>1.35189$ might occur.

Other open questions relate to other subclasses of congestion games. Natural candidates are, e.g., matroid congestion games, generalizing the special case of $k$ uniform matroids we study here. From the viewpoint of matchings, however, also asymmetric versions, where each player has a prescribed demand $k_{i}$ of resources in $R$ are fairly natural.

\section{References}

1. Baruch Awerbuch, Yossi Azar, and Amir Epstein. The price of routing unsplittable flow. In Proc. 37th Annu. ACM Symp. Theory Computing, pages 57-66, 2005.

2. Riccardo Colini Baldeschi, Roberto Cominetti, Panayotis Mertikopoulos, and Marco Scarsini. On the asymptotic behavior of the price of anarchy: Is selfish routing bad in highly congested networks? https://arxiv.org/abs/1703.00927, 2017.

3. Riccardo Colini Baldeschi, Roberto Cominetti, and Marco Scarsini. On the price of anarchy of highly congested nonatomic network games. In Martin Gairing and Rahul Savani, editors, Proc. 9th Int. Symp. Algorithmic Game Theory, volume 9928 of $L N C S$, pages 117-128, 2016.

4. Dietrich Braess. Über ein Paradoxon aus der Verkehrsplanung. Unternehmensforschung, 12:258-0268, 1968. (German).

5. Ioannis Caragiannis, Michele Flammini, Christos Kaklamanis, Panagiotis Kanellopoulos, and Luca Moscardelli. Tight bounds for selfish and greedy load balancing. Algorithmica, 61(3):606-637, 2010.

6. George Christodoulou and Elias Koutsoupias. The price of anarchy of finite congestion games. In Proc. 37th Annu. ACM Symp. Theory Computing, pages 67-73, 2005 . 
7. José R. Correa, Jasper de Jong, Bart de Keijzer, and Marc Uetz. The curse of sequentiality in routing games. In Evangelis Markakis and Guido Schäfer, editors, Proc. 11th Int. Conf. Web and Internet Econ., volume 9470 of LNCS, pages 258271, 2015.

8. Jasper de Jong, Max Klimm, and Marc Uetz. Efficiency of equilibria in uniform matroid congestion games. In Martin Gairing and Rahul Savani, editors, Proc. 9th Int. Symp. Algorithmic Game Theory, LNCS, pages 105-116, 2016.

9. Michal Feldman, Nicole Immorlica, Brendan Lucier, Tim Roughgarden, and Vasilis Syrgkanis. The price of anarchy in large games. In Proc. 48th Annu. ACM Symp. Theory Computing, pages 963-976, 2016.

10. Dimitris Fotakis. Stackelberg strategies for atomic congestion games. ACM Transactions on Computer Systems, 47:218-249, 2010.

11. Elias Koutsoupias and Christos Papadimitriou. Worst-case equilibria. In C. Meinel and S. Tison, editors, Proc. 16th Annu. Sympos. Theoretical Aspects Comput. Sci., volume 1563 of $L N C S$, pages 404-413, 1999.

12. Thomas Lücking, Marios Mavronicolas, Burkhard Monien, and Manuel Rode. A new model for selfish routing. Theoretical Computer Science, 406(3):187-206, 2008.

13. Arthur C. Pigou. The Economics of Welfare. Macmillan, London, UK, 1920.

14. Robert W. Rosenthal. A class of games possessing pure-strategy Nash equilibria. Internat. J. Game Theory, 2(1):65-67, 1973.

15. Tim Roughgarden. The price of anarchy is independent of the network topology. Journal of Computer and System Sciences, 67:341-364, 2002.

16. Tim Roughgarden and Éva Tardos. How bad is selfish routing? Journal of the ACM, 49(2):236-259, 2002.

17. John G. Wardrop. Some theoretical aspects of road traffic research. Proceedings of the Institution of Civil Engineers, 1(3):325-362, 1952. 